EPJ Web of Conferences 41, 04021 (2013)

DOI: $10.1051 /$ epjconf/20134104021

C) Owned by the authors, published by EDP Sciences, 2013

\title{
Interband excitation and carrier relaxation as displacive driving force for coherent phonons
}

\author{
E. M. Bothschafter ${ }^{1,2}$, A. Paarmann ${ }^{3}$, N. Karpowicz ${ }^{2}$, E. S. Zijlstra ${ }^{4}$, M. E. Garcia ${ }^{4}$, \\ F. Krausz ${ }^{1,5}$, R. Kienberger ${ }^{1,2}$, and R. Ernstorfer ${ }^{3}$ \\ ${ }^{1}$ Technical University Munich, Department of Physics, 85748 Garching, Germany \\ ${ }^{2}$ Max Planck Institute for Quantum Optics, 85748 Garching, Germany \\ ${ }^{3}$ Fritz Haber Institute of the Max Planck Society, 14195 Berlin, Germany \\ ${ }^{4}$ University Kassel, Theoretical Physics Department, 34132 Kassel, Germany \\ ${ }^{5}$ Ludwig Maximilian University Munich, Faculty of Physics, 85748 Garching, Germany
}

\begin{abstract}
We report on displacive excitation of large amplitude coherent lattice motions in $\mathrm{TiO}_{2}$ under resonant conditions using sub-6-femtosecond UV pulses. Calculations of non-equilibrium potential energy surfaces reveal a new displacive mechanism for the coherent phonon generation, where a unidirectional displacement of the phonon potential occurs both instantaneously due to carrier excitation and dynamically due to cooling of the hot photo-excited carriers. The carrier cooling dynamics in $\mathrm{TiO}_{2}$ are faster than the phonon period, resulting in an initially anharmonic lattice motion. This effect manifests itself in an effective phase shift of the coherent phonon oscillation, in good agreement between our experiments and the calculations.
\end{abstract}

\section{Introduction}

Transition metal oxides have attracted much attention due to the complex and strong interaction amongst the charge carriers, as well as between carriers and the lattice, such that small perturbations can lead to dramatic modification of the material properties. A typical phenomenon is the significant deformation of the lattice potential following electronic excitations, often studied by displacive excitation of coherent lattice motions by femtosecond optical pulses. Most commonly, the magnitude of the displacive force is assumed to be proportional to the excited charge density [1], and relaxation of the electronic excited state acts to destroy the coherence of the lattice motion.

Here we report on the dynamics of rutile $\mathrm{TiO}_{2}$ after resonant interband UV excitation of electron-hole pairs with <6-femtosecond time resolution. In UV-UV transient reflectivity measurements, we observe a large drop of the reflectivity along with the displacive excitation of the $A_{1}$ phonon at $18 \mathrm{THz}$. Theoretical calculations confirm the displacement of the lattice potential upon laser excitation. As a novel feature, we also observe a significant dependence of the $A_{1}$ potential displacement on the electronic temperature such that the rapid carrier cooling acts as an additional driving force for the coherent phonon. This effect is discussed as a possible cause of the experimentally observed phonon phase shift.

This is an Open Access article distributed under the terms of the Creative Commons Attribution License 2.0, which permits unrestricted use, distribution, and reproduction in any medium, provided the original work is properly cited. 


\section{Methods}

Transient changes in UV reflectivity of a rutile $\mathrm{TiO}_{2}$ (110) surface were measured in a onecolor pump-probe experiment. The UV pulses were generated at $3 \mathrm{kHz}$ repetition rate by third harmonic generation of sub-4-fs, $\sim 780 \mathrm{~nm}$ NIR pulses, focused into a gas cell containing Neon at pressures between 3 and 6 bar [2]. After suppression of the fundamental radiation in a Si periscope, the UV pulses are sent onto a split mirror, one of which can be moved rapidly by a piezo motor, producing a pump and delayed probe beam with a $\sim 3: 1$ intensity ratio. Both beams are focused onto the sample at $\sim 15^{\circ}$ angle of incidence, and the probe intensity is detected as a function of pump-probe delay $\Delta t$. The reflectivity change was detected either directly by rapid delay scanning, or differentially by small-magnitude modulation of the delay and lock-in amplification of the modulated signal component $\Delta R / \Delta t$.

High-accuracy calculations of the potential energy surfaces (PES) for the $A_{1}$ phonon in rutile $\mathrm{TiO}_{2}$ were performed using the WIEN2k density functional theory code. Self-consistent electronic ground state calculations were carried out applying the local density approximation. The excited state properties after laser excitation were obtained by evaluating the changes in the band energies, making use of the Kohn-Sham levels [3]. In particular, our implementation allows independent variation of the number of excited electron-hole pairs and the electronic temperature.

\section{Results and Discussion}

The detected pump-induced UV reflectivity change contains two major features. A remarkably large pump-induced drop of the UV reflectivity of up to $3.5 \%$ was detected, increasing linearly with pump fluence. Secondly, a sinusoidal modulation of the reflectivity with an amplitude of up to $0.3 \%$ was observed with a frequency of $\sim 18 \mathrm{THz}$, allowing the assignment as coherent oscillation of the $\mathrm{A}_{1}$ phonon in $\mathrm{TiO}_{2}$ [4]. Fig. 1 (a) shows a transient of the differential UV reflectivity data $\Delta R / \Delta t$, as well as the least square fits of both above mentioned components, best matched by exponentially decaying oscillations. The best fit results for amplitude, frequency, and phase of the coherent phonon contribution are shown in Fig. 1 (b-d) as a function of incident pump fluence. The amplitude increases linearly with fluence, but frequency and phase are unchanged. Markedly, the initial phase of the oscillation was found to be $\sim 0.8 \pi$, i.e., delayed by $\sim 0.2 \pi$ compared to a cosine oscillation.
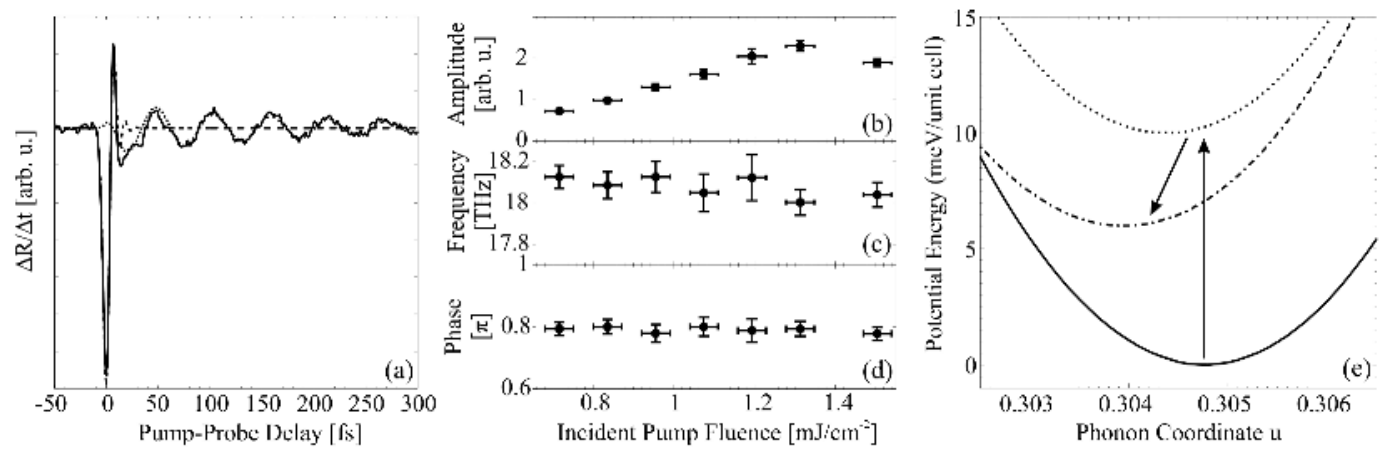

Fig. 1. (a) Differential UV reflectivity change as a function of pump-probe delay (solid line) and fit consisting of two components: The initial, rapid dynamics leading to the overall drop in reflectivity (dashed line) and the coherent phonon oscillation (dotted line). The amplitude (b), frequency (c), and oscillation phase shift (d) are shown as a function of incident pump fluence. (e) Calculations of the potential energy surfaces (PES) of the $\mathrm{A}_{1}$ phonon in $\mathrm{TiO}_{2}$ : ground state (solid line), $1 \mathrm{eV}$-hot excited state (dotted line), and cold excited state (dashdotted line) PES. The curves were shifted vertically for presentation purposes. 
The excitation mechanism of the coherent lattice oscillation observed here is notably different from previous work [4], where an impulsive Raman process via virtual electronic states caused a sine oscillation of the coherent phonon. The resonant interband transition between the valence band with predominant $\mathrm{O}_{2 \mathrm{p}}$ character and the conduction band with dominant $\mathrm{Ti}_{3 \mathrm{~d}}$ character causes an intra-unit cell charge transfer. Thus, one could expect that carrier excitations across the fundamental band gap deform and shift the lattice potential, leading to a displacive excitation of coherent phonons.

This interpretation is strongly supported when comparing the PES along the $A_{1}$ phonon coordinate $u$, calculated for the electronic ground state and the laser-excited states in Fig. 1 (e). Since our UV photon energy of $\sim 5.0 \mathrm{eV}$ exceeds the fundamental $\mathrm{TiO}_{2}$ band gap of $\sim 3.3-3.6 \mathrm{eV}$ [5], we show two curves for laser-excited $\mathrm{TiO}_{2}$ : the PES for the 'hot' electron system (dotted line) with an electronic temperature of $1 \mathrm{eV}$, and the PES for the 'cold' electron system (dashdotted line), both for 0.1 excited electron-hole pairs per unit cell corresponding to our fluence regime. A clear shift of the potential minimum is observed, both between ground state and hot excited state and, with similar magnitude and in the same direction, between the hot and the cold excited state.

The relaxation of highly excited carriers in $\mathrm{TiO}_{2}$ is thought to occur extremely quickly through emission of high frequency phonons [6], predicting a relaxation time of only $\sim 20$ fs for an excess energy of $1 \mathrm{eV}$. Directly measuring these relaxation dynamics is extremely challenging. However, sub-phonon-cycle dynamics of the potential energy surface could be one possible origin of the coherent phonon phase shift observed experimentally, see Fig. 1 (d). We model the atomic motions in the time-dependent PES due to electronic cooling within 20 fs. The resulting trajectory exhibits an oscillation with a delay of $4.6 \mathrm{fs}$ compared to cosine motion, corresponding to a phase shift in good agreement with the experiment. We note, however, that other factors likely also contribute to the experimentally observed phase shift $[1]$.

\section{Conclusions}

We presented experimental and theoretical studies of resonantly excited coherent $A_{1}$ lattice motions in rutile $\mathrm{TiO}_{2}$. Intense Sub-6-fs UV pulses generated in Neon as the $3^{\text {rd }}$ harmonic of sub-4-fs NIR pulses provide the high temporal resolution for detecting the $18.1 \mathrm{THz} \mathrm{A}_{1}$ coherent lattice motion in a transient reflectivity experiment, and determining the phase of the oscillation with high accuracy. Calculations of the non-equilibrium potential energy surfaces reveal displacements of the $A_{1}$ potential minimum. We observed the first indication that not only creation of electron-hole pairs but also the carrier relaxation can constitute a displacive driving force for coherent phonon generation. The carrier cooling dynamics are discussed as a possible cause for the observed phonon phase shift.

\section{References}

1. E. D. M. Riffe and A. J. Sabbah, "Coherent excitation of the optic phonon in Si: Transiently stimulated Raman scattering with a finite-lifetime electronic excitation," Phys. Rev. B 76, 085207 (2007).

2. E. M. Bothschafter, A. Schiffrin, V. S. Yakovlev, A. M. Azzeer, F. Krausz, R. Ernstorfer, and R. Kienberger, "Collinear generation of ultrashort UV and XUV pulses," Opt. Expr. 18, 9173 (2010).

3. M. S. Diakhate, E. S. Zijlstra, and M. E. Garcia, "Quantum dynamical study of the amplitude collapse and revival of coherent $A_{1}$ phonons in bismuth: a classical phenomenon?," Appl. Phys. A 96, 5 (2009).

4. T. Nomoto, A. Sasahara, and H. Onishi, "Optically excited near-surface phonons of $\mathrm{TiO}_{2}(110)$ observed by fourth-order coherent Raman spectroscopy”, J. Chem. Phys. 131, 084703 (2009).

5. L. Chiodo, J. M. García-Lastra, A. Iacomino, S. Ossicini, J. Zhao, H. Petek, and A. Rubio, "Self-energy and excitonic effects in the electronic and optical properties of $\mathrm{TiO}_{2}$ crystalline phases," Phys. Rev. B 82, 045207 (2010).

6. V. P. Zhukov and E. V. Chulkov, "Ab initio approach to the excited electron dynamics in rutile and anatase $\mathrm{TiO}_{2}$," J. Phys.: Condens. Matter 22, 435802 (2010). 